\title{
Automatic identification of route conflict occurrences and their consequences
}

\author{
R. M. P. Goverde, W. Daamen \& I. A. Hansen \\ Transport \& Planning Department, \\ Delft University of Technology, The Netherlands
}

\begin{abstract}
Knowing the source of delays is crucial information to railway infrastructure managers and train operators, but assigning a given delay to its causes is very difficult. Actual delays at stations are monitored online using train describer systems and timetable databases, but it is unknown whether for instance a delay has been caused by a slow running train or by a route conflict where a driver had to slow down due to a restricted signal. In particular, delays due to route conflicts are hard to recognize afterwards, although their identification is crucial to assess and optimize the quality of infrastructure capacity allocation. This paper presents the software tool TNV-Conflict that identifies automatically all signalled route conflicts including the critical sections and conflicting trains. The developed tool can be used offline using train describer logfiles containing chronological infrastructure and train description messages. The object-oriented approach already anticipates an online implementation based on real-time messages. The resulting information is used to analyse the number of route conflicts and their effect on capacity consumption and punctuality, to assign knock-on delays to conflicting trains in a non-discriminatory way, and to identify structural route conflicts corresponding to timetable flaws (e.g. infeasible minimum headways, late trains due to preceding bottlenecks, and early trains due to excessive running time supplements). TNV-Conflict provides essential information to improve capacity allocation and construct reliable train paths. An example application on a railway corridor in the Netherlands illustrates the potential of the tool.
\end{abstract}

Keywords: train delay, train describer, punctuality, route conflict, knock-on delay. 


\section{Introduction}

Railways are usually operated according to a timetable consisting of conflict-free scheduled train paths for all trains running on the network. The actual timetable is usually the result of a detailed planning process up to a year in advance of the actual running, complemented by short-term rescheduling necessitated by disruptions of resources. Nevertheless, the actual train paths always deviate more or less from their schedule due to the stochastic nature of the underlying train running and dwell processes, such as fluctuating driver behaviour, weather conditions, and passenger volumes. Buffer times between train paths prevent that slight schedule deviations instantaneously hinder following trains, but the amount of buffer time depends on the location and is particularly restricted at infrastructure bottlenecks and near transfer stations where connected train lines are synchronized.

A route conflict occurs when the movement authority of a train is restricted by a conflicting preceding train. In practice this implies that a train meets a restricted signal and must prepare to stop before a stop signal protecting an occupied block section. A route conflict always results in running time loss due to braking and re-accelerating, and also additional waiting until the stop signal is cleared if the train comes to a full standstill. The actual time loss depends on rolling stock characteristics, local line speed, and the duration of the stop signal. Moreover, unplanned stops may result in a cascade of route conflicts to successive trains, especially in dense traffic areas. These knock-on delays make up a significant share of all train delays. For example, a case study of the Rotterdam-Dordrecht corridor in the Netherlands revealed that $55 \%$ of the dispunctuality (delays exceeding 3 minutes) is caused by route conflicts [1].

Directive 2001/14/EC [2] gives instructions about transparent infrastructure access conditions and obliges the infrastructure managers to prepare a network statement in order to allocate scarce capacity in a non-discriminatory manner. Performance contracts between the government and train operators - as in Great Britain and the Netherlands - specify some minimum required punctuality and include fines for poor performance. A transparent and objective determination of the cause and responsibility of train delays is a prerequisite for the nondiscriminatory assignment of delays and allocation of fines. Nevertheless, the current delay registration systems can not distinguish between primary and knock-on delays. In the Netherlands, registration of causes of delays is therefore done manually by signallers supported by a monitoring system which displays delays to be explained (delay increase of 3 minutes between two successive stations) and offers a classification tree of delay causes. Hence, next to their primary task of setting routes, the signallers are now also responsible for explaining (larger) train delays. Besides the fact that the signallers themselves can be the cause of delays due to late route setting which might jeopardize the objectivity of their judgment, this extra task may also interfere with the route setting task. In particular, during disruptions the signallers are very busy trying to reduce delays as much as possible rather than spending time to explain train delays. Guided support from the detection systems and automation where 
possible is therefore essential to guarantee a reliable and complete registration of delay causes and responsibilities without excessive work load.

Recognizing knock-on delays from primary delays has also scientific and practical value. Stochastic mathematical models and simulation models of railway operations are based on stochastic distributions of train running times. These distributions should represent the variation of running times with respect to driving behaviour, weather conditions, passenger volumes, etc. Knock-on delays due to route conflicts with other trains are usually computed by the models themselves to evaluate timetable robustness with respect to the primary delays. Hence, these models require stochastic distributions of the primary delays only, while the simulation output should be compared to the total delay including knock-on delays. It is therefore essential that train realization data can be partitioned into unhindered and hindered train runs.

Daamen et al. [3] investigated the existing incident registration systems of the railways in the Netherlands and proposed a conceptual model to automate the identification of route conflicts, the conflicting trains, and the resulting knock-on delays using messages from train describer systems and train detection systems combined with blocking time theory. Elaborating on this, Daamen et al. [4] developed a formal model using a Coloured Petri Net (CPN) to describe the logical and temporal interactions between train number events and the various infrastructure elements and signal blocks. For offline application train describer records can be used which contain all infrastructure and train number messages. Based on the developed formal model a program called TNV-Conflict has been implemented using object-oriented programming in Java, which is also described in Daamen et al. [4].

This paper gives a functional description of TNV-Conflict and illustrates its potential by means of an example using real data. The next section first considers train describer systems (TNV-systems in the Netherlands) and their logfiles, and gives an overview of existing software based on these logfiles. Section 3 presents TNV-Conflict, followed by an example application using real data. The paper ends with conclusions and further developments.

\section{Train describer logfiles}

The key to automatic delay registration is the use of train describers which keep track of train positions based on train numbers and infrastructure messages received from the signalling and interlocking systems [5]. A train number usually steps from one position to another when the train passes a signal. The internal logic of a train describer requires signal, track section and switch information from which it derives the route to the next signal determining the next position. All received infrastructure messages and all generated train number events can be recorded chronologically in train describer logfiles. These records can be used for offline applications such as replaying, punctuality analysis, and infrastructure capacity analysis [6]. The Dutch infrastructure manager ProRail acknowledged the potential of using these train describer records which are therefore centrally archived since March 2000. Dutch train 
describers are called TNV-Systems and the corresponding logfiles are known as TNV-logfiles (TNV is the Dutch abbreviation of Train Number Following).

The TNV-logfiles contain a chronological list of all received infrastructure messages and the train number events generated by the TNV-system itself. A recorded infrastructure message only consists of the date and time, the code of the infrastructure element (section, signal, switch), and a binary state transition (occupied/free, stop/go, left/right), but does not include a train number [6,7].

Goverde [6] developed a method to couple infrastructure messages to train numbers using these TNV-logfiles, which was implemented in the application TNV-Prepare [7]. Thus, TNV-Prepare is able to derive train movements on section level with a precision of a second. An accompanying application, TNV-Filter $[6,8]$, was developed for estimating the remaining running time on the platform track section to/from the stop location by filtering the speed profile of trains over inbound and outbound routes in stations. The application of TNV-Prepare and TNV-Filter enables the estimation of arrival, departure, and through times of trains in stations with an accuracy of several seconds. Recently another application, the VTL-Tool [9], has been developed, which estimates the arrival and departure times based on enhanced TNV-logfiles, called VTL-files. These VTL-files contain extra information on the route used by the train number steps. Additional infrastructure files relate the routes to their infrastructure elements. Thus, the VTL-files allow an automatic derivation of the realized routes of all trains, which is used by the VTL-Tool to automatically derive the detailed realized train paths on section level and estimate all arrival and departure times.

The new application TNV-Conflict is also based on VTL-files combined with infrastructure configuration files which enables a fully automatic matching of train numbers to infrastructure messages over the realized routes. However, TNV-Conflict is extended with signalling logic and accordingly derives the realized blocking times over the successive signal blocks for all trains. This information is used to detect route conflicts and identify the conflicting train numbers. Hence, TNV-Conflict is able to automatically derive route conflicts and resulting knock-on delays, and thus distinguish between primary and knockon delays.

\section{TNV-Conflict}

TNV-Conflict requires the following input to automatically generate all output:

- VTL-files containing the train number and infrastructure messages,

- Infrastructure configuration files including route blocks,

- Timetable files (day plans with scheduled events to compute delays),

- List of train number windows at stations to identify station stops,

- List of section lengths and signal kilometrages (for the visualization).

The first three input files are available from ProRail. The last two items still have to be produced manually based on (digital) infrastructure drawings (OBE and OKT maps) since they are not (yet) available in readable format. 


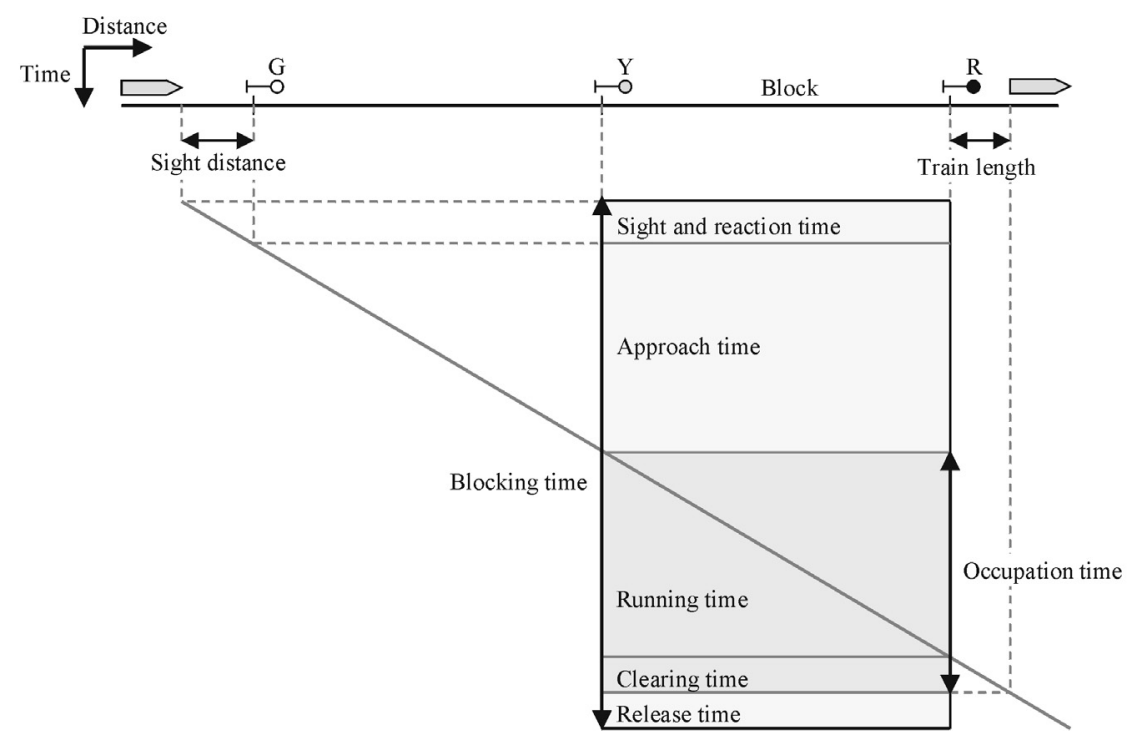

Figure 1: Blocking time of a running train on open track.

TNV-Conflict has been developed using object-oriented programming in Java. Objects are all infrastructure elements (signals, track sections, switches) and signal blocks. The program scans the chronological messages in the VTLfiles and updates the attributes associated to the objects accordingly. For instance, the relevant attributes of a track section in a station layout are its state (occupied, free), last release time and last occupying train number. When a train number steps into a block at a restricted signal then a route conflict in the next block has been detected and the conflicting train number can be found in the critical section of the next block. On open tracks the critical section is just the last track section in the block while in an interlocked route block the critical section is a switch section or the last section whichever has been released last.

However, the logged signal state transitions are binary (stop/go) and so it is unknown whether a signal with state 'go' shows green, yellow, or any other (speed restriction) aspect besides red, unless we also know the state of the signal at the end of the block. Thus, in a simple two-block three-aspect signalling system we have to check the state of both signals at the beginning and the end of a block. If both signals are in the state 'go' then the train entering the block passes a clear block signal. If the first signal has state ' $\mathrm{go}$ ' and the end signal state 'stop' then the train passes an approach signal. And finally, if the first signal shows 'stop' then obviously the train should not have entered the block, so this corresponds to a SPAD (Signal Passed at Danger) incident.

Blocking times and route conflicts can be visualized using blocking time diagrams $[10,11]$. A blocking time is the time interval in which a block section or interlocked route is allocated exclusively to a specific train movement and thus blocked for other trains. On open tracks with automatic block signalling the 




Figure 2: Route conflict visualized by blocking time overlap.

blocking time of a block section by a train consists of the following parts: the sight and reaction time before the approach signal (taken fixed as 12 seconds), the approach time (the running time from the approach signal to the block signal), the running time in the block, the clearing time (running time over the train length to clear the block), and a release time to release the block (taken fixed as 2 seconds), see Figure 1. So, the blocking times exceed the running time over two blocks required for unhindered running. The blocking time of an outbound route block after a scheduled stop in a station does not include an approach time. In an interlocking area with sectional-release route-locking the block is released in steps after clearing the switch sections. For more details, see Pachl [11].

A train experiences hinder when it must brake or wait before a red signal. In three-aspect block signalling, each block signal can act as an approach signal to the signal at the end of the block. When a train passes a yellow signal indicating a red signal at the end of the block then it has to start braking, thus increasing the approach time for the next block. If the block is released by the conflicting train before the train has come to a complete standstill it can accelerate again and move into the block, see Figure 2. Otherwise the train comes to an unscheduled stop and has to wait before the signal until it clears. Because of the braking action both the approach time and the running time may be larger than in the unhindered case as illustrated in Figure 3. In the blocking time diagram a conflict is visualized by an overlap of two blocking times, where of course the hindered 
train can move into the block only after the conflicting train has released the block. So the overlap applies to the approach time (and sight and reaction time) of the hindered train, which is increased to after the block release time of the conflicting train. Note that a blocking time overlap implies a yellow signal at the entrance of the preceding block. Thus, the hinder of a route conflict indicated by a blocking time overlap is mainly experienced in the preceding block, since the approach time of a block is the running time in the previous block.

When a train approaches an interlocked route block to a platform track where it is scheduled to stop, it is not necessary that the block after the departure signal is already set. Hence, a red signal at the end of an inbound route block after the platform (the departure signal) does not imply a route conflict. For this reason the scheduled stops of all trains and a list of all station train number windows must be known. Also the departure from a station after a scheduled stop requires special treatment. In this case, a route conflict occurs when a conflicting route has been set at the requested departure time. The latter is determined as the maximum of the scheduled departure time and the realized arrival time plus a minimum dwell time. If the departure signal is not released at the requested time and a conflicting route after this time is identified then this is a potential route conflict. However, the train may also have been held to secure a transfer with a delayed feeder train, because of a late crew, or any other station process. In these cases, a signaller may set a conflicting route for another train first but this conflict is thus not the cause of a delayed departure. These station processes obviously cannot be detected automatically (except in cases where waiting time regulations apply) and must therefore be registered by the signaller or another person at the control centre. Using the train number and station as key, the registered delay causes can be linked to the delays identified by TNV-Conflict. This way the departure route conflicts can be corrected if another cause has been registered. Likewise, all knock-on delays can be linked to a primary delayed train and its cause as registered in the registration system.

In summary, TNV-Conflict provides the following output:

- The realized event times, delays and delay jumps of all scheduled trains with an accuracy of a few seconds,

- The realized running and dwell times of all trains with conflict indicator,

- The realized platform track sections,

- The route conflicts, conflicting trains, and knock-on delays,

- $\quad$ Linked lists of successive knock-on delays with the primary conflicting trains on top,

- Blocking time diagrams for a given period and corridor.

TNV-Conflict automatically finds the platform track sections where a train has stopped. In particular, when a long platform has multiple track sections TNV-Conflict identifies the track sections on which the head and tail of a train have stopped, thus enabling to use the latest/earliest section entry and release times in the determination of arrival and departure times. The tool has been tested and applied on VTL-files of the two train describer areas The Hague and Rotterdam, including the corridor Leiden-The Hague-Rotterdam-Dordrecht. 


\section{Application}

To illustrate the application of TNV-Conflict we consider two trains running just after midnight from Rotterdam to Dordrecht over the same tracks with threeaspect signalling. Figure 3 shows the realized blocking time diagram. On top of the diagram the route is shown including the tracks sections, signals, and platform tracks. The interlocked routes from signal 938 to just after signal 880 contain switch sections which are denoted by incoming and outgoing tracks. In the figure, the occupation times (running time + clearing time) of the track sections within a block are shown in dark gray while the surrounding blocking times are shown in light gray. Overlapping blocking times are shown in light color within heavy windows. Both trains run from left to right. Blocks are indicated by their signal at the beginning and end, e.g., the first is block 938-898.

The first (upper) train is the regional train R5191 which has scheduled stops at Rotterdam Lombardijen (Rlb) and Barendrecht (Brd). The second train is the (empty) intercity IC72182 which has no scheduled stops but is hindered by the regional train ahead as depicted by the three heavy-windowed overlaps. The IC72182 enters the picture at 00:14:56 in block 938-898 and runs unhindered towards Rotterdam Lombardijen where the R5191 has just departed to block 880-1492. At 00:15:33 the IC72182 enters block 898-880 with a yellow signal 898, while at the same time the R5191 enters block 1492-1472. It takes nine seconds before train R5191 clears the previous block with signal 880 turning yellow. Hence, the approach time of IC72182 to block 880-1492 overlaps with the occupation time of this block by R5191. Therefore, train IC72182 must brake to $40 \mathrm{~km} / \mathrm{h}$. Shortly after signal 880 turns yellow when the R5191 has completely



Figure 3: $\quad$ Example of three successive route conflicts. 
entered block 1492-1472. At 00:16:28 train IC72182 enters block 880-1492 with a yellow signal 880 just before the R5191 enters platform track section 1464BT in Barendrecht (Brd) where it dwells for 30 seconds. Block 1492-1472 includes the platform track at Barendrecht, so the IC72182 cannot enter this block until the R5191 has departed from Barendrecht. Thus, the IC72182 maintains a speed of $40 \mathrm{~km} / \mathrm{h}$ over the entire block 880-1492 and reaches the end of the block 27 seconds after signal 1492 has turned from red to yellow. Therefore, it can continue at $40 \mathrm{~km} / \mathrm{h}$ without stopping and enters block 1492-1472 with again a yellow signal at 00:18:33. After 24 seconds train R5191 clears block 1472-1452, and another 39 seconds later signal 1472 turns green when the R5191 has moved to the next block. The IC is then halfway in block 1492-1472 before Barendrecht and can accelerate again. At 00:20:34 the intercity enters block 1472-1452 with a green signal 1472 and can thus continue at maximum speed again.

In the above example, TNV-Conflict identifies clearly three successive route (or headway) conflicts of IC72182 with the R5191 ahead, including the restrictive signals 898,880 and 1492. If a scheduled through time in Barendrecht is available, the resulting delay can be computed by the difference of the realized through time and the scheduled one. The time loss due to running at a restricted speed between signals 880 and 1472 can be calculated by the delay jump over the through times at Rotterdam Lombardijen and Barendrecht. In practice, there is no reference trajectory available with minimum running times between signals, so that the delay from signal 898 to 1472 cannot be computed. However, the impact of route conflicts on a corridor can be analysed statistically by comparison of similar trains depending on the factor hindered/unhindered. Notice that route conflicts can cause large delays even without an unscheduled stop before a red signal.

\section{Conclusions}

The presented tool TNV-Conflict is based on train describer logfiles (VTL-files) and is therefore suitable to be used for offline analyses. The advanced features of TNV-Conflict enable an effective analysis of route conflicts and their effect on capacity consumption and punctuality, assigning knock-on delays to conflicting trains in a non-discriminatory way, and identifying structural route conflicts corresponding to timetable flaws (e.g. infeasible minimum headways, late trains due to preceding bottlenecks, and early trains due to excessive running time supplements). TNV-Conflict thus provides essential information to improve capacity allocation, construct reliable train paths, discriminate between hindered and unhindered train runs, and validate stochastic models of railway operations. Moreover, it can be used to investigate the need of advanced traffic management systems to predict and resolve route conflicts in areas of heavy traffic.

The object-oriented approach anticipates an online implementation based on real-time infrastructure and train describer messages. The offline tool thus also aims at showing the potential of an online implementation to support an objective incident registration of signallers by providing reliable feedback from the detection systems and automatically link knock-on delays due to conflicting routes. This way signallers can concentrate on route setting and registering direct 
causes of primary train delays. This will result in a more reliable and complete monitoring system with less work load to signallers.

\section{Acknowledgement}

This research has been performed within the TRANSUMO program 'Reliability of transport chains', funded by the Dutch government and in cooperation with ProRail, the Dutch railway infrastructure manager.

\section{References}

[1] Weeda, V.A., Analyse dispunctualiteit, verstoringsregistratie en rij- en halteertijden: Resultaten casestudy Rotterdam-Dordrecht (in Dutch), Report T\&P 2006.007, Department Transport \& Planning, Delft University of Technology, Delft, 2006.

[2] European Commission, Directive 2001/14/EC of the European Parliament and of the Council of 26 February 2001 on the Allocation of Railway Infrastructure Capacity and the Levying of Charges for the Use of Railway Infrastructure and Safety Certification. Official Journal of the European Communities, L75, pp. 29-46, 2001.

[3] Daamen, W., Goverde, R.M.P., Hansen, I.A. \& Weeda, V.A., Monitoring system for reliability of transport chains. Proceedings of the 7th World Congress on Railway Research, Montreal, June 4-8, 2006.

[4] Daamen, W., Goverde, R.M.P. \& Hansen, I.A., Non-Discriminatory Automatic Registration of Knock-On Train Delays. Networks and Spatial Economics, to appear.

[5] Exer, A., Rail Traffic Management. In: Bailey, C. (ed). European Railway Signalling, IRSE, A\&C Black: London, pp. 311-342, 1995.

[6] Goverde, R.M.P., Punctuality of Railway Operations and Timetable Stability Analysis, PhD thesis, Delft University of Technology, 2005.

[7] Goverde, R.M.P. \& Hansen, I.A., TNV-Prepare: Analysis of Dutch railway operations based on train detection data. In: Allan, J., Hill, R.J., Brebbia, C.A., Sciutto, G. \& Sone, S. (eds). Computers in Railways VII, WIT Press: Southampton, pp. 779-788, 2000.

[8] Goverde, R.M.P., Delay Estimation and Filtering of Train Detection Data. Proceedings TRAIL 6th Annual Congress 2000, Part 2, TRAIL Conference Proceedings Series, P2000/3, TRAIL Research School, Delft, 2000.

[9] Stam Van den Berg, B.W.V. \& Weeda, V.A., VTL-Tool: Detailed Analysis of Dutch Railway Traffic. In: Hansen, I.A., Radtke, A., Pachl, J.P. \& Wendler, E. (eds). Proceedings of the 2nd International Seminar on Railway Operations Modelling and Analysis (RailHannover 2007), Hannover, 2007.

[10] Hansen, I.A. \& Pachl, J. (eds), Railway Timetable \& Traffic: Analysis, Modelling, Simulation, Eurailpress: Hamburg, 2008.

[11] Pachl, J., Railway Operation and Control, VTD Rail Publishing: Mountlake Terrace, 2002. 